\title{
Landsat Collections
}

In 2016, the U.S. Geological Survey (USGS) reorganized the Landsat archive into a tiered collection structure. This structure ensures that all Landsat Level-1 products provide a consistent archive of known data quality to support time-series analysis and data "stacking" while controlling continuous improvement of the archive and access to all data as they are acquired. The implementation of collections represents a substantial change in the management of the Landsat archive by ensuring consistent quality through time and across all instruments.

Landsat Collection 1 contains all Level-1 data acquired since 1972 from the Landsat 8 Operational Land Imager (OLI)/Thermal Infrared Sensor (TIRS), Landsat 7 Enhanced Thematic Mapper Plus (ETM+), Landsat 4-5 Thematic Mapper (TM), and Landsat 1-5 Multispectral Scanner (MSS) instruments.

\section{Landsat Collections Tiers}

Landsat Collections consist of three tiered categories: Real-Time, Tier 1, and Tier 2. The Real-Time (RT) tier contains data received from Landsat 8 and Landsat 7 immediately after acquisition. Estimated radiometric and geometric calibration parameters are applied to these scenes, which are made available for download within 12 hours after acquisition. When final radiometric and geometric calibration parameters are calculated (about 16 days for Landsat 8; about 26 days for Landsat 7), the RT scenes are reprocessed with the more precise calibration parameters and placed into either Tier 1 or Tier 2.

Scenes in Tier 1 meet geometric and radiometric quality criteria, which are based on the radial root mean square error (RMSE) value of 12 meters (m) or less. Scenes in Tier 2 have an RMSE value greater than $12 \mathrm{~m}$ and do not meet Tier 1 criteria (fig. 1).

\section{Collection Tier Structure}

The purpose of the collection tier structure is to provide the following:

- Ensure a consistent Landsat archive of known data quality,

- Provide temporary data within 12 hours of acquisition in support of the International Charter to help mitigate the effects of disasters on human life and property,

- Contain the highest quality of inventory and level of processing of Landsat Level-1 products ever created, and

- Support time-series pixel-level analysis and data stacking.

Figure 1. Within 12 hours of acquisition, Landsat 8 and Landsat 7 data are made available for download in the Real-Time tier.
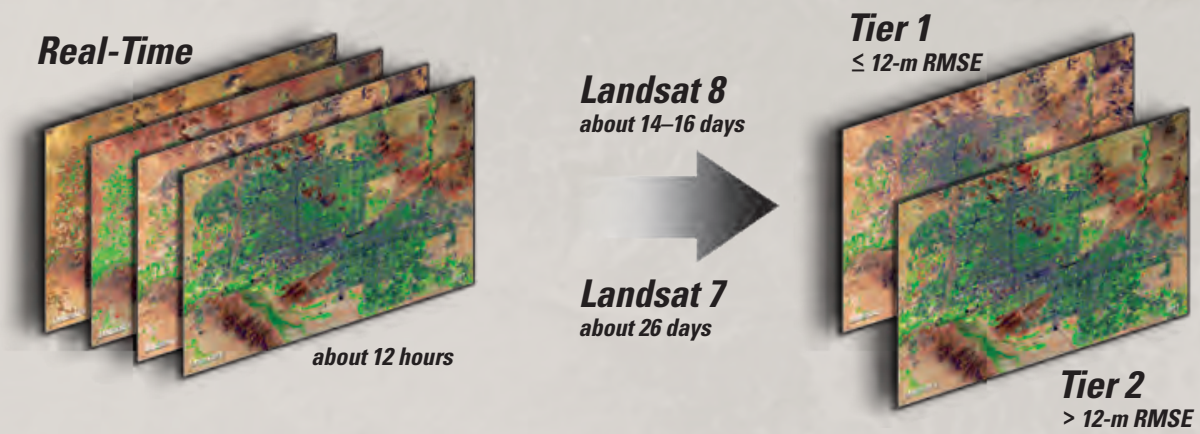
Upon final calculation of the radiometric and geometric parameters, the Real-Time tier acquisitions are reprocessed and assigned into either Tier 1 or Tier 2. 
In order for the user to easily identify which collection tier (RealTime, Tier 1, or Tier 2) a scene is processed into, the Landsat Collection 1 Level-1 product identifier is in accordance with the following naming convention (table 1).

Table 1. An example and explanation of the Landsat Collection 1 product identifier.

[OLI, Operational Land Imager; TIRS, Thermal Infrared Sensor; ETM+, Enhanced Thematic Mapper Plus; TM, Thematic Mapper; MSS, Multispectral Scanner; WRS, Worldwide Reference System; L1TP, Level-1 Precision and Terrain; L1GT, Level-1 Systematic Terrain; L1GS, Level-1 Systematic]

\begin{tabular}{|c|}
\hline Landsat Collection 1 Product Identifier \\
\hline LXSS_LLLL_PPPRRR_YYYYMMDD_yyyymmdd_CC_TX \\
\hline $\mathrm{L}=$ Landsat \\
\hline $\begin{array}{l}\mathrm{X}=\text { sensor ("C"C" = OLI/TIRS combined, "O" = OLI-only, "T" = TIRS-only, } \\
" \mathrm{E} "=\mathrm{ETM}+, " \mathrm{~T} "=\mathrm{TM}, " \mathrm{M} "=\mathrm{MSS})\end{array}$ \\
\hline SS $=$ satellite (“07” = Landsat 7, “08” = Landsat 8$)$ \\
\hline LLLL = processing correction level (L1TP/L1GT/L1GS) \\
\hline $\mathrm{PPP}=\mathrm{WRS}$ path \\
\hline $\mathrm{RRR}=\mathrm{WRS}$ row \\
\hline YYYYMMDD = acquisition year (YYYY)/ month (MM)/ day (DD) \\
\hline yyyymmdd = processing year (yyyy)/ month $(\mathrm{mm}) /$ day $(\mathrm{dd})$ \\
\hline $\mathrm{CC}=$ collection number $(01,02, \ldots)$ \\
\hline $\mathrm{TX}=$ collection category ("RT" = Real-Time, "T1" = Tier 1, "T2" = Tier 2) \\
\hline Examples \\
\hline
\end{tabular}

LC08_L1GT_029030_20151209_20160131_01_RT

LE07_L1TP_016039_20040918_20160211_01_T1

LT04_L1GS_017036_19821115_20160315_01_T2

LM01_L1GS_017039_19760131_20160225_01_T2

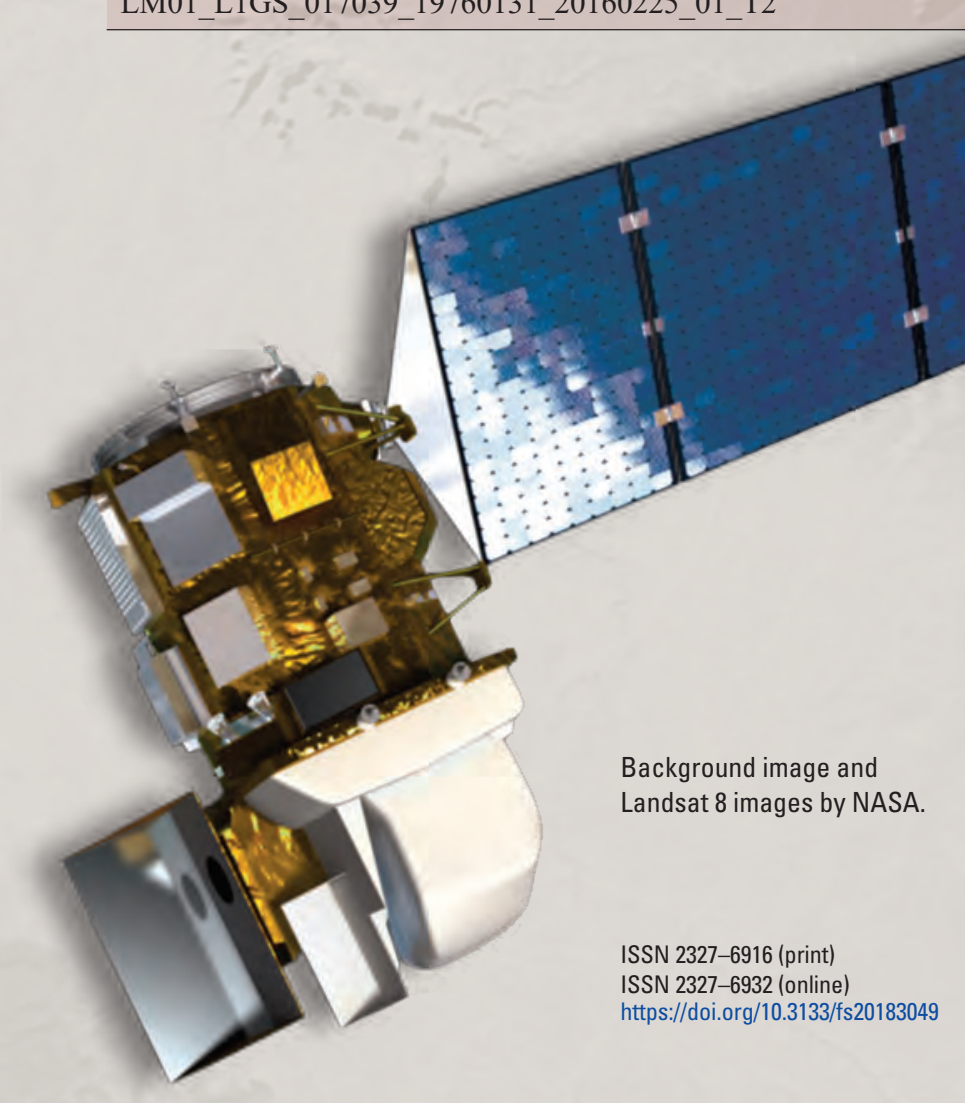

\section{Additional Landsat Collection 1 Processing Changes}

Along with the Landsat product identifier, the following files and processing changes are also delivered with all Collection 1 Level-1 data products:

- New Quality Assessment (QA) bands and Angle Coefficient Files

- Additional metadata (MTL.txt) fields: Saturation Bits field, Land Cloud Cover assessment

- New cloud cover algorithm: C Function of Mask (CFMask)

- Specific for Landsat 8 OLI/TIRS: integration of the TIRS stray light correction

- Specific for Landsat 4-5 TM: no- Correction Data (PCD) scenes

- Calibration Parameter File (CPF) file name includes the collection number to uniquely link CPFs to corresponding Landsat collections

- Response Linearization Look Up Tables (RLUTs) name changes (Landsat 8 only): RLUTs are files that accompany CPFs and contain a look up table

Landsat Collection 1 data are available for download at no charge from EarthExplorer at https://earthexplorer.usgs.gov/, GloVis at https://glovis.usgs.gov/, and LandsatLook Viewer at https://landsatlook.usgs.gov/.

For additional information or to learn more about the Landsat Collections inventory structure, please visit the Landsat Collections web page at https:// www.usgs.gov/land-resources/nli/ landsat/landsat-collections.

Visit https://www.usgs.gov for more information about the USGS and https://remotesensing.usgs.gov for specifics about the Land Remote Sensing Program.

\section{For More Information}

Questions about Landsat operations, data products, and data access can be directed to

Landsat User Services

U.S. Geological Survey

Earth Resources Observation and Science (EROS) Center 47914 252nd Street

Sioux Falls, SD 57198

Phone: 605-594-6151 or 1-800-252-4547

Email: custserv@usgs.gov

Twitter:@USGSLandsat

Facebook: https://www.facebook.com/NASA.Landsat 